\title{
BMJ Open Risk factors for severity of COVID-19: a rapid review to inform vaccine prioritisation in Canada
}

\author{
Aireen Wingert (D) , Jennifer Pillay, Michelle Gates (D), Samantha Guitard, \\ Sholeh Rahman, Andrew Beck (D) , Ben Vandermeer, Lisa Hartling
}

To cite: Wingert A, Pillay J, Gates M, et al. Risk factors for severity of COVID-19: a rapid review to inform vaccine prioritisation in Canada. BMJ Open 2021;11:e044684. doi:10.1136/ bmjopen-2020-044684

- Prepublication history and additional online supplemental material for this paper are available online. To view these files, please visit the journal online (http://dx.doi.org/10. 1136/bmjopen-2020-044684)

Received 09 September 2020 Revised 16 December 2020 Accepted 21 April 2021

Check for updates

(c) Author(s) (or their employer(s)) 2021. Re-use permitted under CC BY-NC. No commercial re-use. See rights and permissions. Published by BMJ.

Alberta Research Centre for Health Evidence, Department of Pediatrics, University of Alberta Faculty of Medicine and Dentistry, Edmonton, Alberta, Canada

Correspondence to

Aireen Wingert;

awingert@ualberta.ca

\section{ABSTRACT}

Objectives Rapid review to determine the magnitude of association between potential risk factors and severity of COVID-19, to inform vaccine prioritisation in Canada.

Setting Ovid MEDLINE(R) ALL, Epistemonikos COVID-19 in L.OVE Platform, McMaster COVID-19 Evidence Alerts and websites were searched to 15 June 2020. Eligible studies were conducted in high-income countries and used multivariate analyses.

Participants After piloting, screening, data extraction and quality appraisal were performed by a single experienced reviewer. Of 3740 unique records identified, 34 were included that reported on median 596 (range 44-418794) participants, aged 42-84 years. 19/34 (56\%) were good quality.

Outcomes Hospitalisation, intensive care unit admission, length of stay in hospital or intensive care unit, mechanical ventilation, severe disease, mortality.

Results Authors synthesised findings narratively and appraised the certainty of the evidence for each risk factor-outcome association. There was low or moderate certainty evidence for a large ( $\geq 2$-fold) magnitude of association between hospitalisation in people with COVID-19, and: obesity class III, heart failure, diabetes, chronic kidney disease, dementia, age $>45$ years, male gender, black race/ethnicity (vs non-Hispanic white), homelessness and low income. Age $>60$ and $>70$ years may be associated with large increases in mechanical ventilation and severe disease, respectively. For mortality, a large magnitude of association may exist with liver disease, Bangladeshi ethnicity (vs British white), age $>45$ years, age $>80$ years (vs 65-69 years) and male gender among 20-64 years (but not older). Associations with hospitalisation and mortality may be very large ( $\geq 5$-fold) for those aged $\geq 60$ years.

Conclusions Increasing age (especially $>60$ years) may be the most important risk factor for severe outcomes. High-quality primary research accounting for multiple confounders is needed to better understand the magnitude of associations for severity of COVID-19 with several other factors.

PROSPERO registration number CRD42020198001.

\section{INTRODUCTION}

COVID-19 is an infectious respiratory disease caused by the newly identified SARS-CoV-2, ${ }^{1}$ which reached worldwide pandemic status in

\section{Strengths and limitations of this study}

- This rapid review captured a broad range of risk factors and outcomes associated with COVID-19 severity.

- Eligible studies reported independent associations through statistical adjustment and were applicable to high-income countries.

- The certainty of evidence was assessed for each risk factor-outcome-population association.

- The rapid approach involved pilot testing each review step with multiple reviewers until a high level of agreement was achieved; then a single experienced reviewer completed study selection, data extraction and risk of bias assessments.

- The review includes studies published up to June 2020; guidance on vaccine prioritisation should also consider emerging evidence.

early March $2020 .^{2}$ As of 7 December, there were over 65.8 million confirmed cases worldwide and 1.5 million deaths attributed to the virus. ${ }^{3}$ Most people who develop COVID-19 will experience mild-to-moderate illness primarily affecting the respiratory system and recover at home. ${ }^{4}$ In more severe cases, patients may require specialised care (eg, admission to hospital and/or intensive care unit (ICU), assisted ventilation $)^{5}$ as the disease can progress to respiratory failure and/or affect multiple organ systems. ${ }^{4}$

Given the rapid spread of COVID-19 since its first emergence in late 2019, and potential for severe illness (including death), the development of a preventive vaccine has become a global priority. ${ }^{6}$ Vaccine development has been progressing at an unprecedented pace $^{7-10}$; however, the initial vaccine supply is not expected to be sufficient to cover the entire population right away. Therefore, it is of high priority to policymakers to plan for the efficient, effective and equitable allocation of vaccines when limited supply will necessitate recommendations for the vaccination of certain groups earlier than others. 
Due to the novel nature of COVID-19, these groups for early vaccination have not yet been established. ${ }^{11}$

The National Advisory Committee on Immunisation (NACI) is an expert advisory body that provides advice on the use of vaccines in Canada. ${ }^{12}$ At the time of writing, NACI is developing guidance on priority pandemic immunisation strategies for COVID-19 vaccination when initial vaccine supply is limited. ${ }^{11}$ To inform this guidance, NACI is using its recently published Ethics, Equity, Feasibility and Acceptability (EEFA) Framework ${ }^{13}$ to ensure these factors are systematically and comprehensively considered. One of the evidence informed tools that make up this framework is the 'Equity Matrix' which has adapted the PROGRESS-Plus model of health determinants and outcomes ${ }^{14}$ to ensure important vaccine-specific equity factors are explicitly included. The resulting ' $\mathrm{P}^{2} \mathrm{ROG}$ RESS And Other Factors' framework includes a range of biological and social factors that likely contribute to inequities in health outcomes across population groups (eg, pre-existing disease/condition, place/state of residence, race/ethnicity/culture/language, occupation, gender identity/sex), but it is not yet clear how each factor might apply to COVID-19 outcomes. A discussion on the use of this Equity Matrix, with evidence from this rapid review, as a critical tool to guide the ethically just allocation of scarce resources is published elsewhere. ${ }^{15}$

With the aim of providing timely, evidence-informed guidance on pandemic vaccine prioritisation, NACI required a rigorous and expedited synthesis of the available evidence on population groups that are at increased risk of severe illness and mortality as a result of COVID-19. Responding to this need, we conducted a rapid review to determine the magnitude of association between ' $P{ }^{2} R O G$ RESS And Other Factors' and risk of severe outcomes of COVID-19.

\section{METHODS}

\section{Review approach}

Rapid reviews are a form of knowledge synthesis that accelerate the process of conducting a traditional systematic review through streamlining or omitting some steps to produce evidence in a resource-efficient manner. ${ }^{16}$ Methods for streamlining one or more stages of the review process are highly dependent on context such as the organisational capacity of the review producer (eg, trained and experienced personnel), and needs of policy-makers for decision making ${ }^{17}$; one or more of the systematic review dimensions (ie, scope, comprehensiveness, rigour/quality control, approach to synthesis, conclusions) may be modified for a rapid review. ${ }^{17}$

The need for empiric evidence to inform the prioritisation of pandemic immunisation strategies in Canada necessitated a rapid but rigorous approach to synthesising the currently available data. Therefore, we performed a rapid review informed by traditional systematic review methodology, ${ }^{19}$ with several modifications to allow for the evidence to be synthesised on an expedited timeline. We sought stakeholder input on the review question, eligibility criteria and outcomes, to inform the protocol for applicability and feasibility. We used a single experienced reviewer to select studies, extract data and assess risk of bias, whereas in traditional systematic reviews these steps typically involve two reviewers to some degree. To ensure methodological rigour, we conducted pilot testing with more than one reviewer at each step; once a high level of agreement was achieved, a single reviewer proceeded with completing the step. In addition, we focused the scope of the review to include only higher quality studies (ie, using adjusted analysis), and those having high applicability to Canada (eg, high-income countries with universal-like healthcare systems).

NACI's High Consequence Infectious Disease Vaccine Working Group was consulted to develop and refine the scope of the review (ie, priority population(s), risk condition(s)/factor(s), and outcomes of interest), but was not involved in the conduct of the review. The working group was not involved in selection of studies nor the synthesis of findings.

The review was conducted following an a-priori protocol (PROSPERO \#CRD42020198001). Because there is not yet formal guidance on the reporting of rapid reviews, reporting adheres to the Preferred Reporting Items for Systematic Reviews and Meta-Analyses. ${ }^{20}$

\section{Literature search}

A health sciences librarian searched Ovid MEDLINE(R) ALL on 15 June 2020 using concepts related to COVID19, P²ROGRESS And Other Factors, and severe outcomes (online supplemental file 1). The search was limited to studies published in English or French in 2020. Additionally, the search was limited to populations in countries that are members of the Organisation for Economic Cooperation and Development (OECD) ${ }^{21}$ in an effort to include studies of highest relevance to the Canadian context. Editorials and letters were excluded. We supplemented the Medline search by handsearching Epistemonikos COVID-19 in L.OVE Platform (https://app. iloveevidence.com/topics) and McMaster COVID-19 Evidence Alerts (https://plus.mcmaster.ca/COVID-19/) for relevant prognosis or aetiology studies up to 12 June 2020. A handsearch of relevant websites recommended by the NACI working group was also undertaken, as well as continual surveillance for publication of relevant preprints located by the search. Searches were exported to an Endnote Library (X9, Clarivate Analytics, Philadelphia, Pennsylvania USA) and duplicates removed.

\section{Eligibility criteria}

We included studies published in English or French since 1 January 2020 that reported on the magnitude of association between potential P ${ }^{2}$ ROGRESS And Other Factors and several outcomes of COVID-19 (online supplemental file 2). Eligible source populations, in order of priority, were people (1) from a general/community sample, (2) with COVID-19 confirmed (by laboratory 
testing or epidemiologic linkage) and (3) hospitalised with COVID-19. Although considered potentially of interest, studies only including people with a risk factor of interest were not included. We excluded studies where the entire study population had severe disease (eg, ICU settings). To ensure relevance to the Canadian context, studies had to be conducted in OECD countries ${ }^{21}$; we included OECD studies from countries that do not provide universal (or near universal) coverage for core medical services (ie, Chile, Greece, Mexico, Poland, the Slovak Republic and the USA) ${ }^{22}$ but considered these to be less applicable to the Canadian context when interpreting the findings. The infection must have been confirmed by laboratory testing or linked epidemiologically (eg, household contact). Studies including populations with other pandemic-related infections (eg, severe acute respiratory syndrome, Middle East respiratory syndrome) were excluded if data specific to COVID-19 cases could not be isolated.

The exposures of interest were any $\mathrm{P}^{2}$ ROGRESS And Other Factors believed to be associated with differential outcomes across population groups (ie, pre-existing conditions, place or state of residence, race/ethnicity/ culture/language, immigration, refugee status, occupation, gender identity or sex, religion or belief system, education or literacy level, socioeconomic status, social capital, age and other factors). ${ }^{23}{ }^{24}$ We did not include as risk factors any signs or symptoms on presentation with COVID-19. Eligible comparators were those within the same source population (eg, all hospitalised, as described above) that did not have the $\mathrm{P}^{2}$ ROGRESS And Other Factor, or experienced a P2ROGRESS And Other Factor to a different degree (eg, older vs younger). We excluded studies of interventions.

Any length of follow-up for outcomes of interest was acceptable. Eligible studies reported on at least one primary outcome (ie, rate of hospitalisation, hospital length of stay, severe disease (as defined by study authors; eg, composite outcome of ICU transfer or death), ICU admission and length of stay, need for mechanical ventilation (MV), and mortality (case fatality or all-cause). We refer to this range of outcomes as 'severe COVID-19' or 'severity of COVID-19' throughout the review, though distinct from the composite outcome of 'severe disease'. Each of these outcomes are applicable to at least one of the above-mentioned eligible populations. In order to prioritise the most rigorous and applicable evidence, we included only prospective and retrospective cohort studies that employed a multivariate analysis and provided results of the independent contribution of $\mathrm{P}^{2}$ ROGRESS And Other Factors to severe outcomes, while accounting for potential confounders (minimally age and sex). Preprints were included only if they were accepted by a peer-reviewed journal; preprints that were later published (between the date of the search and manuscript submission) were included. Government reports from handsearched websites were eligible.

\section{Study selection}

All records retrieved by the searches were exported to a Microsoft Office Excel (Microsoft, Redmond, Washington, USA) spreadsheet for screening. After piloting the eligibility criteria on a sample of 70 records, one reviewer independently screened records for inclusion by title/abstract, and those deemed to be potentially relevant were assessed by full text. Uncertainties about the inclusion of any full text study were resolved through consultation with a second reviewer.

\section{Data extraction}

Following a pilot round, one reviewer independently extracted data from each included study into an Excel workbook. We extracted data on (1) population size and demographics, (2) setting, (3) dates of data collection, (4) COVID-19 ascertainment method, (5) coinfections, (6) outcomes reported with definitions for composite outcomes (eg, severe disease), (7) number of participants analysed and (8) relevant outcome data related to $\mathrm{P}^{2} \mathrm{ROG}-$ RESS factors of interest (using the most adjusted model, if more than one was reported). For both continuous and dichotomous outcomes, we extracted adjusted relative effect sizes (ie, OR, risk ratio (RR), HR) and measures of variability $(95 \% \mathrm{CI})$. A second reviewer was consulted in the event of uncertainty about any of the extracted data. Given the expedited approach, we extracted only data that were reported within the included studies and made no attempt to contact authors for missing or unclear data.

\section{Quality assessments}

To expedite quality assessments, we did not use a formal tool; instead we focused on key variables that were considered to be most relevant to the topic, and that would allow for meaningful stratification of studies by quality. The key variables that we used to assess the quality of the included studies were (1) the extent of adjustment for relevant covariates (ie, basic adjustment for age and sex, vs more extensive adjustment for numerous potential confounders including comorbidities), (2) follow-up duration and extent of censorship for some outcomes (eg, $\geq 2$ weeks for mortality) and (3) inappropriate or large exclusions from the study and/or analysis (eg, missing data on risk factor status or analytical variables). Following assessment of these key variables by a single reviewer, studies without concerns for all three criteria were rated good while others were rated fair. A second reviewer was consulted in the case of uncertainty about the assessment of any individual study.

\section{Synthesis}

Given substantial clinical (eg, risk factors and/or comparators examined, outcome definitions) and methodological (varying covariates included in the adjusted analyses, different measures of association) heterogeneity, it was not thought appropriate to pool the studies statistically. Instead, we present a narrative summary of the results across studies for each risk factor. When making conclusions about the association between a P²ROGRESS And Other Factor and an 
outcome, we focused primarily on the magnitude of effect rather than statistical significance, which is heavily dependent on sample size. We categorised associations to be small/ unimportant $(\mathrm{OR}$ or $\mathrm{RR}) \leq 1.70)$, moderate (1.71 to 1.99$)$, large $(\geq 2.00)$ or very large $(\geq 5.00) .{ }^{25}$ When determining the magnitude, we compared findings across all relevant studies and often relied heavily on the findings of the largest and/or good-quality studies.

\section{Certainty of evidence}

The expedited approach to evidence synthesis did not allow for a formal appraisal of the certainty of evidence across studies for each P2ROGRESS And Other FactorOutcome association. Instead, a single reviewer assessed the certainty of the evidence for each association considering relevant components of the Grading of Recommendations Assessment, Development and Evaluation (GRADE) approach ${ }^{2627}$ : (1) directness in terms of country (presence of universal healthcare) and source population (community sample vs hospitalised patients), (2) sample size $(\mathrm{n}<500$ considered small $)$ and magnitude of association, (3) study quality and (4) consistency of associations (in direction and magnitude) across studies. Bodies of evidence started at high certainty ${ }^{28}$ and were rated down for weaknesses in any of the aforementioned characteristics. The level of certainty in associations are referred to using the terms 'uncertain' (no or very low certainty), 'may' (low or some certainty) and 'probably' (moderate certainty). ${ }^{29}$ At least two other reviewers confirmed the certainty of evidence appraisals, with disagreements resolved by discussion.

\section{RESULTS}

\section{Characteristics of studies}

Of 3740 unique records identified by the searches, 949 were screened at full text, and 34 studies that reported on 32 unique populations were included in the review (figure 1, online supplemental file 3) shows studies excluded by full text, with reasons). ${ }^{30-63}$ Three studies conducted in the $\mathrm{UK}^{465154}$ used overlapping cohorts from a single medical/research database and were considered as a single population in the analysis. Another large UK study $^{63}$ is likely to also be overlapping with these populations, but the degree of overlap is not known.

Table 1 shows the characteristics of the included studies (full details about individual studies in online supplemental file 4). The studies were published between 23 April and 6 July 2020, and half $(17 / 34,50 \%)$ reported on populations in the USA. $^{30} 31383943-4547-50525356586061$ The remaining countries represented (Italy, ${ }^{32}$ 34-37 425762 Spain, ${ }^{33}$ $\mathrm{UK}^{40465154555963}$ ) all have universal or universal-like healthcare (one study used data from 17 countries). All studies reported on adults, and the overall median was 596 participants (range 44-418794). The mean or median age of the populations studied ranged

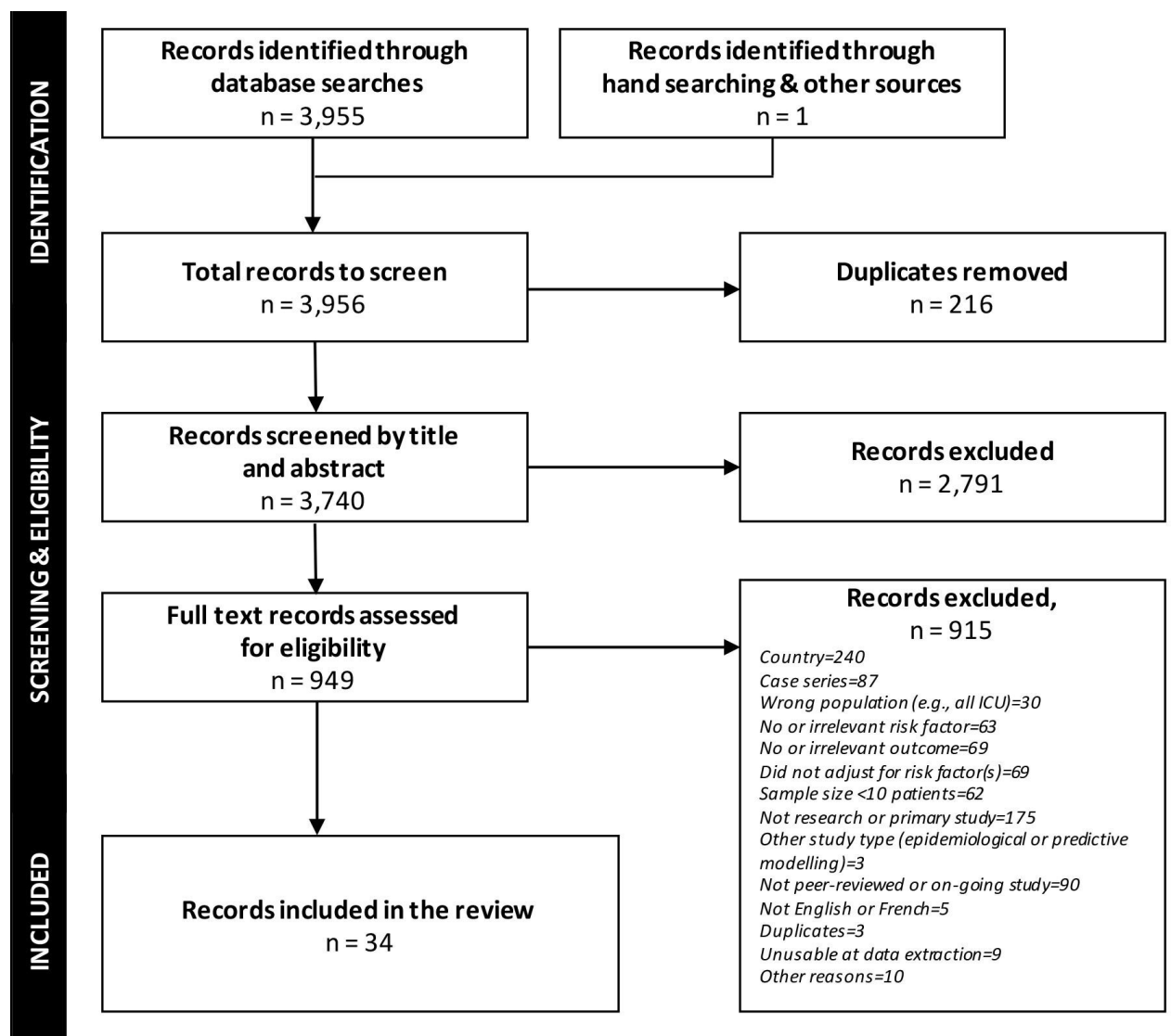

Figure 1 PRISMA flow of study selection. PRISMA, Preferred Reporting Items for Systematic Reviews and Meta-Analyses. 
Table 1 Included studies overview $(n=34)$

\begin{tabular}{|c|c|c|c|c|}
\hline $\begin{array}{l}\text { Study design and } \\
\text { country } \\
\text { (noof studies) }\end{array}$ & $\begin{array}{l}P^{2} R O G R E S S \text { risk factors } \\
\text { (no of studies*) }\end{array}$ & $\begin{array}{l}\text { COVID-19 } \\
\text { (no of studies) }\end{array}$ & $\begin{array}{l}\text { Primary outcomes } \\
\text { (no of studies*) }\end{array}$ & $\begin{array}{l}\text { Risk of } \\
\text { bias } \\
\text { (no of } \\
\text { studies) }\end{array}$ \\
\hline
\end{tabular}

*A study may contribute to more than one risk group or outcome.

†Study of healthcare workers includes data from Australia, Canada, Chile, China, Germany, India, Ireland, Italy, Netherlands, New Zealand, Pakistan, Poland, Singapore, South Africa, Sweden, UK and USA.

‡Severe disease, defined by studies as (number of studies): requiring high-flow oxygen (1); ICU or MV (1); non-invasive ventilation or MV (1); MV (4); ICU or mortality (composite)(4); hospitalisation and/or 30-day mortality (composite)(1); MV or mortality (composite)(1); ICU, MV, discharge to hospice or death (composite)(1).

COPD, chronic obstructive pulmonary disease; ICD, International Classification of Diseases; ICU, intensive care unit; MV, mechanical ventilation; NR, not reported; RT-PCR, reverse transcription PCR.

from 42 to 84 years (in $32 / 34(94 \%)$ mean age was $54-71$ years). Most studies (16/34, 47\%) examined the association between risk factors and outcomes in a hospitalised population. Studies reported variable definitions of 'severe disease'; we considered them sufficiently similar to be grouped under this outcome. Studies most commonly reported on the independent association of pre-existing conditions ( $n=27$ studies), gender identity or sex $(n=18)$ and race or ethnicity $(n=12)$ with severe outcomes (most commonly hospitalisation, $n=9)$. $\mathrm{P}^{2}$ ROGRESS And Other Factors not examined in the included studies were immigration or refugee status, religion or belief system, social capital and substance abuse disorders. There were also no data specific to pregnant women, indigenous populations, people with disabilities, nor different ages in children.

\section{Study quality}

The majority of studies $(19 / 34,56 \%)$ were rated as good quality $^{3031363840-434749525355-6062}$ because they adjusted for age, sex and pre-existing disease in their analysis, had adequate follow-up of outcomes and few or no missing data. The remaining studies had flaws in one or more of the three domains that we considered to be most important for this review. 


\section{Association between risk factors and outcomes}

Table 2 shows a summary of findings for associations between each reported risk factor and outcomes of interest; detailed data are in online supplemental file 5 .

There was low or moderate certainty of evidence for important/large associations with increased hospitalisation in people having confirmed COVID-19, for the following risk factors: obesity class III (body mass index $\geq 40 \mathrm{~kg} / \mathrm{m}^{2}$; one study, $\mathrm{n}=5297$ ), ${ }^{56}$ heart failure (two studies, $\mathrm{n}=6331$ ), ${ }^{30} 56$ diabetes (two studies, $\mathrm{n}=6331$ ), ${ }^{30} 56$ chronic kidney disease (confirmed COVID-19 or community sample; two studies, $\mathrm{n}=424073),{ }^{54}$ dementia (one study, $\mathrm{n}=418794$ ), ${ }^{54}$ age over 45 years (vs 45 or younger; two studies, $\mathrm{n}=6331$ ), ${ }^{30}$ male gender (three studies, $\mathrm{n}=3812$ ), ${ }^{30} 5658$ black race/ethnicity (vs non-Hispanic white; confirmed COVID-19 and community samples, 5 studies in four populations, $\mathrm{n}=428606), 3051545658$ homelessness (one study, $\mathrm{n}=1052)^{30}$ and low income ( $<25$ th vs $>50$ th percentile; one study, $\mathrm{n}=1052) .{ }^{30}$ Age over 60 and over 70 years may be associated with important increases in the rate of MV (one study, $\mathrm{n}=486)^{47}$ and severe disease (one study, $\mathrm{n}=2725),{ }^{56}$ respectively.

There may be important associations for increased mortality with liver disease (two studies, $\mathrm{n}=20597),{ }^{40} 60$ Bangladeshi ethnicity (vs British white; one study, $\mathrm{n}=130091$ ), ${ }^{63}$ and age over 45 years (vs <45 years; three studies, $n=87819) .^{405663}$ The data were somewhat inconsistent for gender, with most studies showing moderate certainty of no important effect, but one large fair quality study $(n=130091)^{63}$ from the UK that stratified its analysis by age showed that hospitalised males aged 20-64 years (but not older) may be at about twofold increased risk of mortality compared with females.

Associations with hospitalisation and mortality may be very large for those aged over 60 years (two studies, $\mathrm{n}=6331$ for hospitalisation ${ }^{30}{ }^{56}$; three studies, $\mathrm{n}=24163$ for mortality ${ }^{40}{ }^{56}$ ) and are probably very large for those over 70 years (two studies, $\mathrm{n}=6331$ for hospitalisation $^{3056}$; two studies, $n=22858$ for mortality ${ }^{40} 56$ ). One study ( $\mathrm{n}=63094)^{63}$ directly compared subgroups of older hospitalised adults, showing that compared with those aged 65-69 years, there may be no important association with mortality among adults aged 70-79 years, but the strength of associations may increase about a magnitude of twofold for those 80 years and older. Studies treating age on a continuum or across small increments consistently found that the magnitude of association for hospitalisation and mortality increased with increasing age (eg, approximately $2 \%-6 \%$ and $5 \%-10 \%$ relative increase per year) (three studies in two populations, $\mathrm{n}=422275$ for hospitalisation ${ }^{515458} ; 11$ studies, $\mathrm{n}=6877$ for mortality). ${ }^{32-343842455253555862}$

A moderate magnitude of association may exist between MV (four studies, $\mathrm{n}=1559$ ), ${ }^{45} 474953$ ICU admission (two studies, $\mathrm{n}=873$ ), ${ }^{45} 49$ and severe disease (one study, $\mathrm{n}=2725)^{56}$ and obesity (body mass index $\geq 30$ or $40 \mathrm{~kg} / \mathrm{m}^{2}$ ); severe disease and heart failure (one study, $\mathrm{n}=2725)^{56}$; mortality and haematological malignancy (one study, $\mathrm{n}=1183)^{59}$; MV and male gender (four studies, $\mathrm{n}=881)^{34474953}$; and hospitalisation and social deprivation (highest vs lowest quintile; one study, $\mathrm{n}=340996){ }^{51}$

There was moderate certainty evidence for no important increase in hospitalisation with chronic respiratory conditions (four studies in three populations, $\mathrm{n}=425125),{ }^{30} 515456$ cardiovascular disease apart from heart failure (ie, coronary artery disease, hypertension, hyperlipidaemia; four studies in three populations, $\mathrm{n}=425125),{ }^{30} 515456$ non-specific cancer (two studies, $\mathrm{n}=6331),{ }^{3056}$ Asian race/ethnicity other than Bangladeshi (vs non-Hispanic white; 3 studies in two populations, $\mathrm{n}=424073$ ), 515456 and current or former smoking (five studies in three populations, $n=425125) . .^{3046515456}$ Additionally, there was moderate certainty evidence for no important increase in severe disease with chronic respiratory conditions (one study, $\mathrm{n}=2725),{ }^{56}$ chronic kidney disease (two studies, $\mathrm{n}=2922),{ }^{31} 56$ non-specific cancer (two studies, $\mathrm{n}=2769$ ), ${ }^{36} 56$ and black race/ethnicity (vs non-Hispanic white; two studies, $\mathrm{n}=3030)^{43} 56$ and no important increase in mortality with obesity (body mass index $\geq 30 \mathrm{~kg} / \mathrm{m}^{2}$; six studies, $\mathrm{n}=8716$ ), 424550535658 chronic respiratory conditions (four studies, $\mathrm{n}=23315$ ), 38405356 diabetes (four studies, $\mathrm{n}=23315$ ), 38405356 chronic kidney disease (three studies, $\mathrm{n}=23058$ ), nonspecific cancer (three studies, $\mathrm{n}=24041),{ }^{40} 5659$ male gender (nine studies, $\mathrm{n}=27875$ ), ${ }^{32-34} 384042535658$ Black (five studies, $\mathrm{n}=135418)^{4555565863}$ or Asian race/ethnicity (vs nonHispanic white; three studies, $\mathrm{n}=4015)^{455556}$ and social deprivation (lowest vs highest quintile; one study, $\mathrm{n}=130091) .{ }^{63}$ Overall, there were few data for the ICU and MV outcomes.

\section{DISCUSSION}

Responding to a need for empiric evidence to inform decision making on Canada's immunisation strategies, ${ }^{11}$ in this rapid review, we synthesised studies employing multivariate analysis to ascertain potential independent associations between 'P2 ROGRESS And Other Factors' and severe outcomes of COVID-19. Among 22 potential risk factors examined across the included studies, the most important risk factors (ie, those associated with large/important increased risk or odds; RR or OR $\geq 2.0$ ) for hospitalisation among those with confirmed COVID-19 were several pre-existing chronic health conditions (obesity class III, heart failure, diabetes, chronic kidney disease [community sample or with COVID-19], dementia [community sample]), older age ( $>45$ years vs younger), male gender, black race/ethnicity (community sample or with COVID-19), homelessness and low income ( $\leq 25$ th vs $>50$ th percentile). Liver disease may be have a large magnitude of association with increased mortality among people with COVID-19; advancing age ( $>45$ years vs younger) and Bangladeshi ethnicity (vs British white) are likely to have large magnitude of associations with increased mortality among hospitalised patients. There is evidence to suggest that male gender may be associated 
Table 2 Summary of evidence for associations between risk factors and severe outcomes of COVID-19

Magnitude of association (confidence in association)‡, by outcome Magnitude of associations are shown as: uncertain (no/very low confidence), no important association (-; OR or RR $\leq 1.70)$, moderate association (+1.71-1.99), large/important association $(++; \geq 2.00)$, or very large important association $(+++; \geq 5.00)$

Risk factor (at-risk vs ICU Mechanical Severe reference population)*

Population†

Hospitalisation

admission ventilation

disease

Mortality

\section{Pre-existing conditions}

Body mass index (BMI) $\left(\mathrm{kg} / \mathrm{m}^{2}\right) \S$

Underweight $(<18.5)$ vs Hospitalised normal (18.5-24.9)

\begin{tabular}{|c|c|c|c|c|}
\hline & $\begin{array}{l}- \\
\text { (low) }\end{array}$ & $\begin{array}{l}- \\
\text { (low) }\end{array}$ & & $\begin{array}{l}- \\
\text { (low) }\end{array}$ \\
\hline $\begin{array}{l}- \\
\text { (low) }\end{array}$ & uncertain & uncertain & $\begin{array}{l}- \\
\text { (low) }\end{array}$ & $\begin{array}{l}- \\
\text { (low) }\end{array}$ \\
\hline $\begin{array}{l}+ \\
\text { (low) }\end{array}$ & $\begin{array}{l}+ \\
\text { (low) }\end{array}$ & $\begin{array}{l}+ \\
\text { (low) }\end{array}$ & (low) & $\begin{array}{l}- \\
\text { (moderate) }\end{array}$ \\
\hline $\begin{array}{l}++ \\
\text { (low) }\end{array}$ & & uncertain & $\begin{array}{l}+ \\
\text { (low) }\end{array}$ & $\begin{array}{l}- \text { to }+ \\
\text { (low) }\end{array}$ \\
\hline
\end{tabular}

normal

Community

normal

sample or

positive for

COVID-19

Obesity class I and II

Community

$(\geq 30.0)$ vs normal

sample or

positive for

COVID-19

Obesity class III ( $\geq 40.0$ ) vs Positive for

Respiratory conditions

Chronic, varied (eg,

asthma, COPD)

COVID-19

Community sample or positive for COVID-19

Prior pneumonia

Community sample

(moderate)

uncertain uncertain

(moderate) (moderate)

(low)

Cardiovascular disease

Heart failure

Community

sample

Positive for

COVID-19

Coronary artery

Community

disease, hypertension,

sample or

hyperlipidaemia,

positive for

composite outcomes

COVID-19

Diabetes

Community

sample

Positive for

COVID-19

Liver disease

Positive for

COVID-19

Hospitalised

Chronic kidney disease

Community sample or positive for COVID-19

Inflammatory bowel disease Positive for COVID-19

Dementia/chronic neurological disorders

Alzheimer's disease or Community dementia

sample
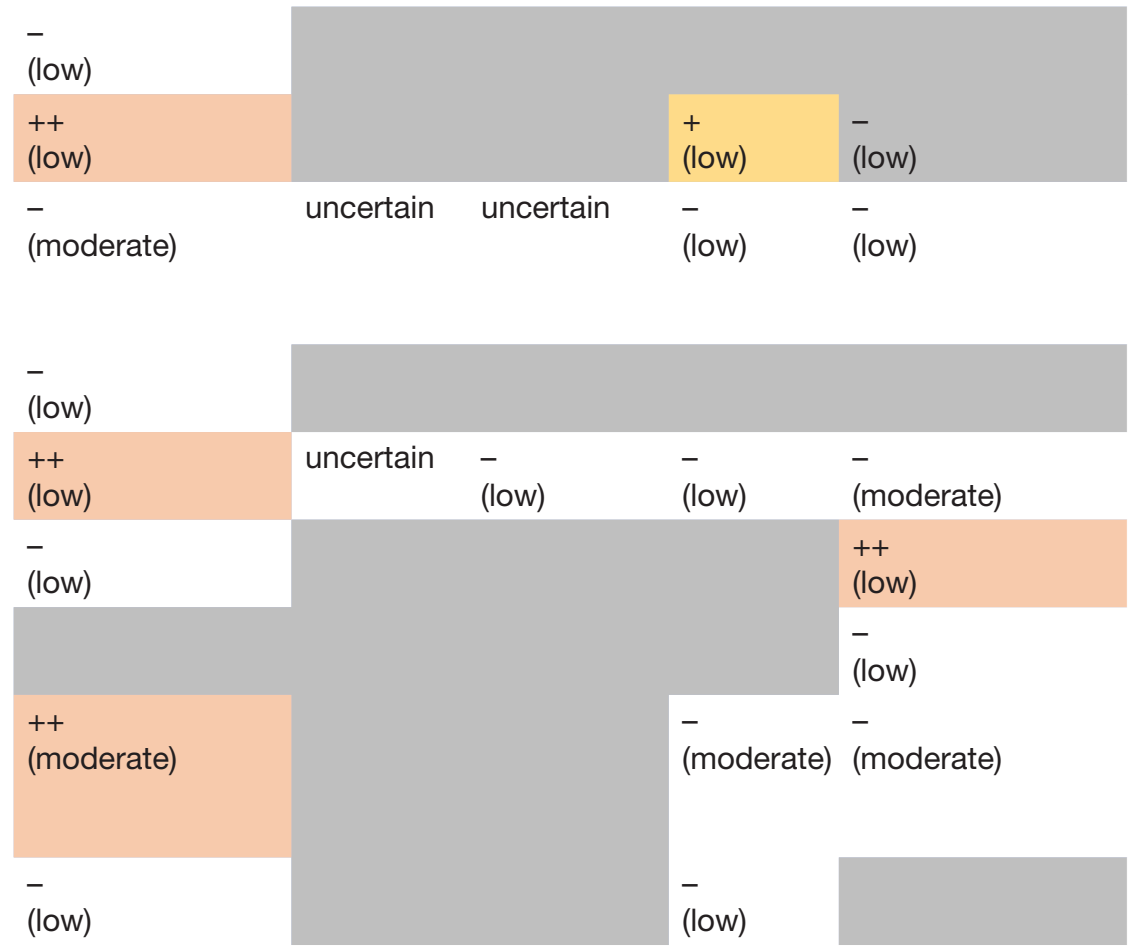

$++$

(low) 
Table 2 Continued

Risk factor (at-risk vs reference population)*

Chronic neurological disorders

\section{Cancer}

Any cancer

Haematological

malignancy

Immunocompromised

Rheumatic disease

HIV

Mental health

Depression

Ever visited a psychiatrist

Positive for COVID-19

Positive for COVID-19

Positive for COVID-19

Positive for COVID-19

Hospitalised

Community sample

\section{Other factors}

Age§

$45-54$ vs $\leq 45$ years old

Positive for COVID-19

$50-64$ vs $\leq 45$ years old

Positive for COVID-19

$>60$ vs $\leq 45$ years old

Positive for COVID-19

$>70$ or 75 vs $\leq 45$ years old Positive for COVID-19

$>80$ vs $\leq 45$ years old

Positive for COVID-19

70-79 vs 65-69 years old

Hospitalised

>80 vs 65-69 years old Hospitalised

Increased age Community

(continuous/incremental) $\uparrow$ sample or positive for COVID-19

Gender or sex

Male vs female (all ages, Community mean 54-73)

Male vs female (20-64 years) $^{\star \star}$ sample

Positive for COVID-19

Hospitalised Race/ethnicity

Magnitude of association (confidence in association)‡, by outcome Magnitude of associations are shown as: uncertain (no/very low confidence), no important association (-; OR or RR $\leq 1.70$ ), moderate association ( $+1.71-1.99)$, large/important association $(++\geq 2.00)$, or very large important association $(+++; \geq 5.00)$

ICU Mechanical Severe

Hospitalisation admission ventilation disease

Mortality (low)

$$
\text { (moderate) }
$$$$
\text { (moderate) } \text { (moderate) }
$$

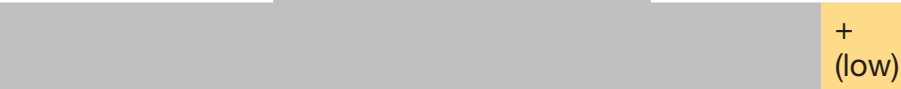

$$
\begin{aligned}
& \text { uncertain } \\
& \\
& - \\
& \text { (low) } \\
& - \\
& \text { (low) }
\end{aligned}
$$$$
\text { uncertain }
$$

uncertain

uncertain
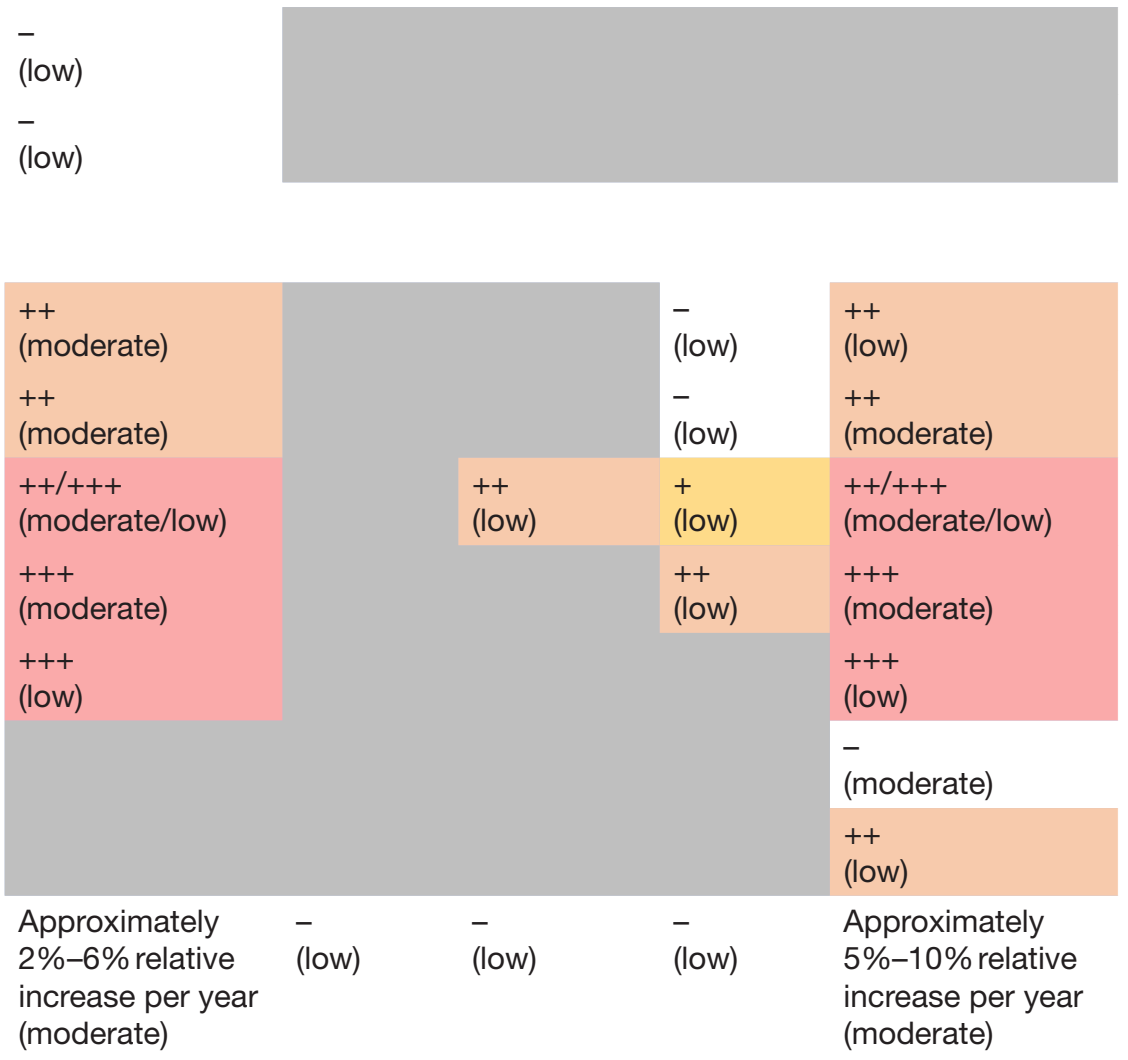

(low)

$++$

(moderate)

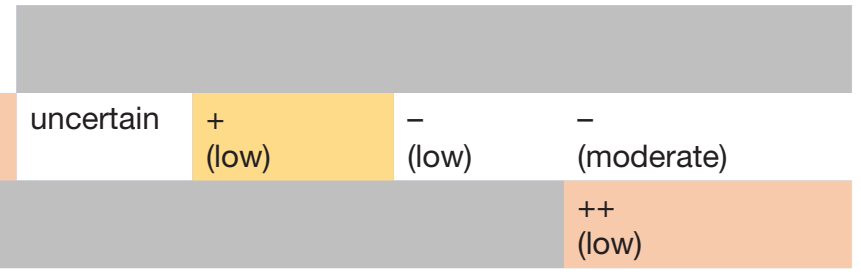

Continued 
Risk factor (at-risk vs reference population)*

Black vs non-Hispanic white British white white

Asian vs white
Magnitude of association (confidence in association)‡, by outcome Magnitude of associations are shown as: uncertain (no/very low confidence), no important association (-; OR or RR $\leq 1.70)$, moderate association ( $+1.71-1.99)$, large/important association $(++\geq 2.00)$, or very large important association $(+++; \geq 5.00)$

ICU Mechanical Severe

Hospitalisation admission ventilation disease Mortality

sample or

positive for

COVID-19

Hispanic vs non-Hispanic

Culture/language/immigrant/ refugee status
Positive for COVID-19

Community sample or positive for COVID-19

Hospitalised
$++$

(low)

(m

$-$

(low) admission ventilation (moderate) (moderate) (moderate) uncertain (low) (low) $-$ (low) (low) (low) $-$ (moderate)
Place of residence/household size

Living in a low income area

Positive for COVID-19

Homeless vs has a home

Positive for COVID-19

Suburban vs urban

Hospitalised hospital

1, 3 or 4 vs 2 household members

Community sample

Occupation

Laryngologist or intubator vs assistant

Education level

Lower education vs university degree

Socioeconomic status

Highest vs lowest quintile of social deprivation Income $\leq 25$ th vs $>50$ th or 75th percentile

$\geq$ Average vs below average income

\section{Smoking}

Current or former vs never Community

COVID-19
patients sample or positive for COVID-19

Community sample

Community sample

Positive for COVID-19

Community sample

(low)

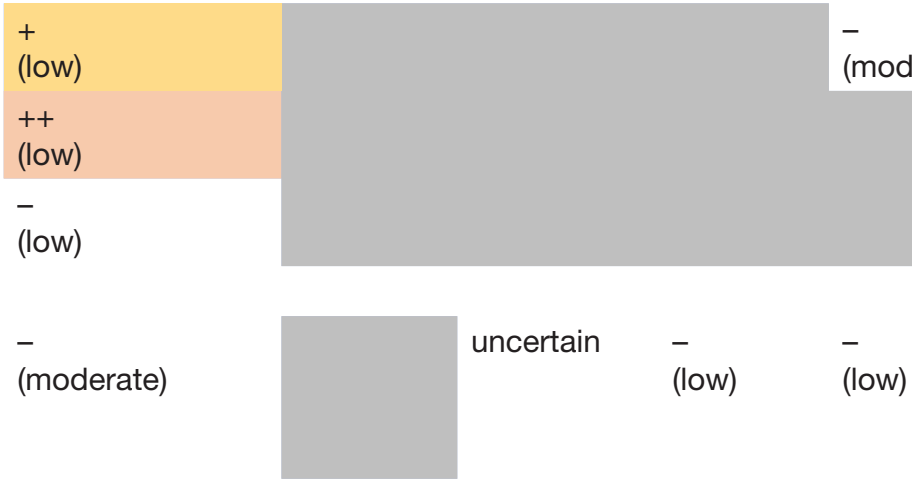

uncertain

(low)

(low)
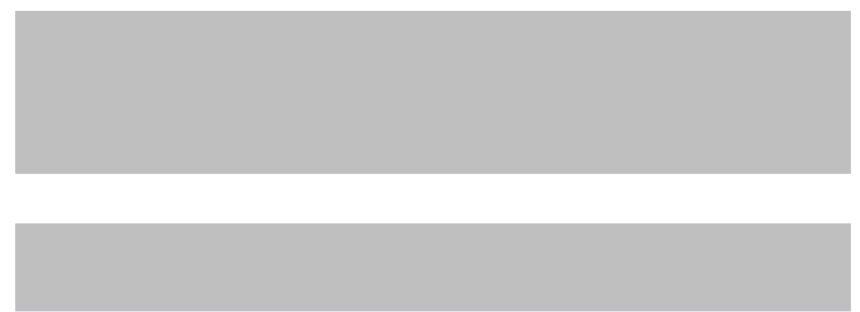

(moderate)

(low)
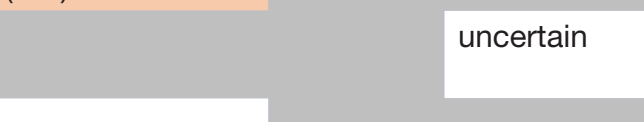

(

w) 


\begin{tabular}{|c|c|c|c|c|c|c|}
\hline \multirow[b]{2}{*}{$\begin{array}{l}\text { Risk factor (at-risk vs } \\
\text { reference population)* }\end{array}$} & \multirow[b]{2}{*}{ Population† } & \multicolumn{5}{|c|}{$\begin{array}{l}\text { Magnitude of association (confidence in association) } \ddagger \text {, by outcome } \\
\text { Magnitude of associations are shown as: uncertain (no/very low } \\
\text { confidence), no important association (-; OR or } R R \leq 1.70) \text {, moderate } \\
\text { association ( }+1.71-1.99 \text { ), large/important association }(++; \geq 2.00) \text {, or very } \\
\text { large important association }(+++; \geq 5.00)\end{array}$} \\
\hline & & Hospitalisation & $\begin{array}{l}\text { ICU } \\
\text { admission }\end{array}$ & $\begin{array}{l}\text { Mechanical } \\
\text { ventilation }\end{array}$ & $\begin{array}{l}\text { Severe } \\
\text { disease }\end{array}$ & Mortality \\
\hline Above vs within guidelines & $\begin{array}{l}\text { Community } \\
\text { sample or } \\
\text { positive for } \\
\text { COVID-19 }\end{array}$ & $\begin{array}{l}- \\
(\text { low })\end{array}$ & & & & \\
\hline \multicolumn{7}{|l|}{ Physical activity level } \\
\hline Below vs within guidelines & $\begin{array}{l}\text { Community } \\
\text { sample or } \\
\text { positive for } \\
\text { COVID-19 }\end{array}$ & $\begin{array}{l}- \\
\text { (low) }\end{array}$ & & & & \\
\hline
\end{tabular}

*When not listed, the reference group are those without the risk factor.

†Outcomes of severe disease (as defined by authors), ICU admission, mechanical ventilation and mortality are all in a hospitalised population, except for liver disease, where findings differed depending on the population denominator used.

$\ddagger A$ formal assessment of the quality/confidence of the evidence was not performed but was informed by the Grading of Recommendations, Assessment, Development and Evaluations (GRADE) approach. We determined our confidence in the magnitude of the associations by considering primarily study limitations (risk of bias), consistency in findings across studies and precision (sample size). Very low confidence indicates that were have no/very low confidence about possible associations; low means that the evidence indicates that there may be an association; moderate means that the evidence indicates that there probably is an association. High certainty evidence was not found for any association.

§For categorical data for age, and BMI, the reference group differed slightly across studies.

IFor continuous or incremental data for age, the rate of hospitalisation and mortality outcomes, approximately half of the studies analysed data on a continuum (with the remainder reporting in incremental categories, for example, 5 years units).

${ }^{* *}$ Subgroup data from one study that analysed the younger population separately.

COPD, chronic obstructive pulmonary disease; ICU, intensive care unit; RR, risk ratio.

with increased mortality among younger (20-64 years), but not older men.

Among the factors that increase the chance of severe outcomes, age seemed to be the most influential; adults older than 60 years may have at least five times the magnitude of association with hospitalisation and mortality from COVID-19 compared with those aged less than 45 years. This association with increased hospitalisation and mortality appears to magnify at least to some degree even for those older than 60 years, with those aged over 80 years possibly having double the magnitude of association for mortality of those aged 65-69 years.

The findings of this rapid review will be used to populate the Equity Matrix of NACI's EEFA Framework, ${ }^{13}$ which will be a part of a suite of considerations for informing the development of NACI recommendations on priority pandemic immunisation strategies when initial COVID-19 vaccine supply is limited. NACI will be using the results of this rapid review and their current understanding of the epidemiology of COVID-19 in Canada to identify distinct inequities associated with COVID-19, potential reasons for these inequities and suggested interventions to reduce inequities and improve access to vaccine when it becomes available. The Equity Matrix applied to COVID-19 with evidence to date can be found elsewhere. ${ }^{15}$

\section{Limitations of the evidence}

There are several limitations to the evidence base. Though we focused the review on better quality studies that minimally controlled for age and sex, the strength of certain associations should be interpreted cautiously because there are likely to be multiple unmeasured confounders that have not been accounted for. For example, studies reporting on associations between outcomes and age did not adjust for nursing home residency and studies examining race did not account for occupation which may be an important confounder influencing susceptibility to the infection. ${ }^{63}$ In addition, it is important to be aware that criteria for COVID-19 testing and hospitalisation may differ by place and time, but it is difficult to predict how this may have impacted the findings. In general, many studies conducted testing based on symptoms and the evidence is likely most applicable to these populations. The evidence for MV, ICU admission and severe disease outcomes was relatively sparse. As we located no evidence meeting our publication date and inclusion criteria to inform the impact of immigration or refugee status, religion or belief system, social capital, substance abuse disorders, pregnancy, Indigenous identity, living with a disability, 
nor differing levels of risk among children in various age groups, there is a need for high quality primary research (accounting for multiple confounders) to better understand the magnitude of association with these risk factors. Given the rapid emergence of new evidence on the topic, potential associations (or lack of association) for which only low or very low certainty of evidence is available should continue to be reviewed as new primary research is published.

\section{Strengths and limitations of the review}

Our analysis across a large range of risk factors by detailed outcomes along the continuum of the natural history of COVID-19 disease highlights the methodological rigour and comprehensiveness of the present work. Whereas many rapid reviews omit all assessment of study quality and certainty of the evidence, we felt this was critical for rigour of interpretation and undertook these steps. Given our rapid approach, it is possible that studies were missed and that undetected errors in data exist. We mitigated this by piloting the screening and data extraction process and using experienced reviewers, and it is unlikely that any important studies were missed that would have altered the findings of the review. ${ }^{64}$ We conducted risk of bias assessments at the study level, rather than at the outcome level, and incorporated these into our GRADE assessments at the risk factor-outcome-population level. Given that our eligibility criteria narrowed inclusion to higher quality studies that were most applicable to the review objective, it is unlikely that our appraisals of the certainty of evidence would be substantially impacted. Nevertheless, an in-depth evaluation of the study quality at the outcome level may be worth undertaking if feasible in future work.

The evidence presented in this review should be interpreted as most applicable to people with COVID-19 symptoms or in general populations, but not necessarily to those with severe infections because studies focused solely on patients with severe COVID-19 (ie, in ICU settings) were excluded. Most studies of patients in the ICU setting that we located were relatively small and descriptive in nature, such that many would have been otherwise excluded, due to lack of adjustment, or only have been able to provide low or very low certainty evidence due to their lack of precision. Additionally, generalisations to other countries should be made with caution, as high-risk groups in these populations may differ.

Acknowledgements We would like to thank the National Advisory Committee on Immunisation (NACl) High Consequence Infectious Disease Vaccine Working Group (Caroline Quach, Shelley Deeks, Yen Bui, Kathleen Dooling, Robyn Harrison, Kyla Hildebrand, Michelle Murti, Jesse Papenburg, Robert Pless, Nathan Stall, and Stephen Vaughan) for their contributions to the project. We also thank Liz Dennett (MLIS) for conducting the Medline search, and Karyn Crawford for assisting with article retrieval.

Contributors AW, JP, AB, BV and LH contributed to the conception and design of the study. AW, JP, SG, SR, AB and BV contributed to the screening of eligible studies. $A W, S G, S R$ and $A B$ contributed to the acquisition of data. AW, JP, MG, BV and LH contributed to the synthesis and interpretation of data. AW, JP and MG drafted the manuscript. AW, JP, MG, SG, SR, AB, BV and LH revised the manuscript for important intellectual content. All authors approved the manuscript for submission.

Funding National Advisory Committee on Immunisation (Canada); contract No. 4600001536 .

Competing interests All authors have completed the ICMJE uniform disclosure form at www.icmje.org/coi_disclosure.pdf and declare: grants from the National Advisory Committee for Immunisation during the conduct of the study; no other relationships or activities that could appear to have influenced the submitted work. LH is supported by a Canada Research Chair in Knowledge Synthesis and Translation.

Patient consent for publication Not required.

Provenance and peer review Not commissioned; externally peer reviewed.

Data availability statement All data relevant to the study are included in the article or uploaded as online supplemental information. No additional data available; all data used in this review are available within the manuscript and accompanying online supplemental files.

Supplemental material This content has been supplied by the author(s). It has not been vetted by BMJ Publishing Group Limited (BMJ) and may not have been peer-reviewed. Any opinions or recommendations discussed are solely those of the author(s) and are not endorsed by BMJ. BMJ disclaims all liability and responsibility arising from any reliance placed on the content. Where the content includes any translated material, BMJ does not warrant the accuracy and reliability of the translations (including but not limited to local regulations, clinical guidelines, terminology, drug names and drug dosages), and is not responsible for any error and/or omissions arising from translation and adaptation or otherwise.

Open access This is an open access article distributed in accordance with the Creative Commons Attribution Non Commercial (CC BY-NC 4.0) license, which permits others to distribute, remix, adapt, build upon this work non-commercially, and license their derivative works on different terms, provided the original work is properly cited, appropriate credit is given, any changes made indicated, and the use is non-commercial. See: http://creativecommons.org/licenses/by-nc/4.0/.

\section{ORCID iDs}

Aireen Wingert http://orcid.org/0000-0002-8024-5221

Michelle Gates http://orcid.org/0000-0002-9941-9981

Andrew Beck http://orcid.org/0000-0002-8308-2202

Lisa Hartling http://orcid.org/0000-0001-8341-3991

\section{REFERENCES}

1 World Health Organization. Naming the coronavirus disease (COVID-19) and the virus that causes it, 2020. Available: https:// www.who.int/emergencies/diseases/novel-coronavirus-2019/ technical-guidance/naming-the-coronavirus-disease-(covid-2019)and-the-virus-that-causes-it [Accessed 31 Jul 2020].

2 World Health Organization. Coronavirus disease 2019 (COVID-19): situation report - 51. Geneva, Switzerland: World Health Organization, 2020. https://www.who.int/docs/default-source/ coronaviruse/situation-reports/20200311-sitrep-51-covid-19.pdf

3 World Health Organization. Coronavirus disease 2019 (COVID-19): weekly epidemiological update 17. Geneva, Switzerland: World Health Organization, 2020. https://www.who.int/publications/m/item/ weekly-epidemiological-update-8-december-2020

4 Rodriguez-Morales AJ, Cardona-Ospina JA, Gutiérrez-Ocampo $\mathrm{E}$, et al. Clinical, laboratory and imaging features of COVID-19: a systematic review and meta-analysis. Travel Med Infect Dis 2020;34:101623.

5 Government of Canada. Coronavirus disease 2019 (COVID-19): epidemiology update. Ottawa, Canada: Government of Canada, 2020. https://health-infobase.canada.ca/src/data/covidLive/ Epidemiological-summary-of-COVID-19-cases-in-Canada-Canada. ca.pdf

6 World Health Organization. Accelerating a safe and effective COVID-19 vaccine. Geneva, Switzerland: World Health Organization, 2020. https://www.who.int/emergencies/diseases/novel-coronavirus2019/global-research-on-novel-coronavirus-2019-ncov/acceleratinga-safe-and-effective-covid-19-vaccine

7 Health Canada. Statement on UK's authorization of its first COVID-19 vaccine, manufactured by Pfizer/BioNTech. Ottawa, Canada: Health Canada, 2020. https://www.canada.ca/en/health-canada/news/2020/ 12/statement-on-uks-authorization-of-its-first-covid-19-vaccinemanufactured-by-pfizerbiontech.html 
8 Health Canada. Vaccines and treatments for COVID-19: progress. Ottawa, Canada: Health Canada, 2020. https://www.canada.ca/en/ public-health/services/diseases/2019-novel-coronavirus-infection/ prevention-risks/covid-19-vaccine-treatment.html

9 World Health Organization. Draft landscape of COVID-19 candidate vaccines. Geneva, Switzerland: World Health Organization, 2020. https://www.who.int/publications/m/item/draft-landscape-of-covid19-candidate-vaccines

10 Thanh Le T, Andreadakis Z, Kumar A, et al. The COVID-19 vaccine development landscape. Nat Rev Drug Discov 2020;19:305-6.

11 Government of Canada. Research priorities for COVID-19 vaccines to support public health decisions. Ottawa, Canada: Government of Canada, 2020. https://www.canada.ca/en/public-health/services/ immunization/national-advisory-committee-on-immunization-naci/ research-priorities-covid-19-vaccines.html

12 Government of Canada. National Advisory Committee on immunization (NACl): membership and representation. Ottawa, Canada: Government of Canada, 2020. https://www.canada.ca/en/ public-health/services/immunization/national-advisory-committeeon-immunization-naci/naci-membership-representation.html

13 Ismail SJ, Hardy K, Tunis MC, et al. A framework for the systematic consideration of ethics, equity, feasibility, and acceptability in vaccine program recommendations. Vaccine 2020;38:5861-76.

14 Evans T, Brown $\mathrm{H}$. Road traffic crashes: operationalizing equity in the context of health sector reform. Inj Control Saf Promot 2003;10:11-12

15 Ismail SJ, Tunis MC, Zhao L. Navigating inequities: a roadmap out of the pandemic. BMJ 2020.

16 Hamel C, Michaud A, Thuku M, et al. Defining rapid reviews: a systematic scoping review and thematic analysis of definitions and defining characteristics of rapid reviews. J Clin Epidemiol 2021:129:74-85.

17 Hartling L, Guise J-M, Kato E, et al. A taxonomy of rapid reviews links report types and methods to specific decision-making contexts. J Clin Epidemiol 2015;68:1451-62.

18 Tricco AC, Langlois EV, Straus SE, eds. Rapid reviews to strengthen health policy and systems: a practical guide. Geneva, Switzerland: World Health Organization, 2017.

19 Higgins J, Thomas J, Chandler J. Cochrane handbook for systematic reviews of interventions. 2nd Edition. Chinchester, UK: John Wiley \& Sons, 2019.

20 Liberati A, Altman DG, Tetzlaff J, et al. The PRISMA statement for reporting systematic reviews and meta-analyses of studies that evaluate healthcare interventions: explanation and elaboration. $B M J$ 2009;339:b2700.

21 Organisation for Economic Cooperation and Development. Member countries, 2020. Available: https://www.oecd.org/about/membersand-partners/ [Accessed 24 Aug 2020].

22 Fraser Institute. Comparing performance of universal health care countries, 2019. Available: https://www.fraserinstitute.org/studies/ comparing-performance-of-universal-health-care-countries-2019 [Accessed 24 Aug 2020].

23 O'Neill J, Tabish H, Welch V, et al. Applying an equity lens to interventions: using PROGRESS ensures consideration of socially stratifying factors to illuminate inequities in health. J Clin Epidemiol 2014;67:56-64.

24 Oliver S, Kavanagh J, Caird J. Health promotion, inequalities and young people's health: a systematic review of research. London, UK: EPPI-Centre, Social Science Research Unit, Institute of Education, University of London, 2008.

25 Guyatt GH, Oxman AD, Sultan S, et al. GRADE guidelines: 9. Rating up the quality of evidence. J Clin Epidemiol 2011;64:1311-6.

26 Guyatt G, Oxman AD, Akl EA, et al. GRADE guidelines: 1. Introduction-GRADE evidence profiles and summary of findings tables. J Clin Epidemiol 2011;64:383-94.

27 Murad MH, Mustafa RA, Schünemann $\mathrm{HJ}$, et al. Rating the certainty in evidence in the absence of a single estimate of effect. Evid Based Med 2017;22:85-7.

28 Iorio A, Spencer FA, Falavigna M, et al. Use of GRADE for assessment of evidence about prognosis: rating confidence in estimates of event rates in broad categories of patients. BMJ 2015;350:h870.

29 Santesso N, Glenton C, Dahm P, et al. GRADE guidelines 26: informative statements to communicate the findings of systematic reviews of interventions. J Clin Epidemiol 2020;119:126-35.

30 Azar KMJ, Shen Z, Romanelli RJ, et al. Disparities in outcomes among COVID-19 patients in a large health care system in California. Health Aff 2020;39:1253-62.

31 Bhargava A, Fukushima EA, Levine M. Predictors for severe COVID-19 infection. Clin Infect Dis 2020.
32 Bianchetti A, Rozzini R, Guerini F, et al. Clinical presentation of COVID19 in dementia patients. J Nutr Health Aging 2020;24:560-2.

33 Borobia A, Carcas A, Arnalich F, et al. A cohort of patients with COVID-19 in a major teaching hospital in Europe. J Clin Med 2020;9:1733. doi:10.3390/jcm9061733

34 Busetto L, Bettini S, Fabris R, et al. Obesity and COVID-19: an Italian snapshot. Obesity 2020;28:1600-5.

35 Cecconi M, Piovani D, Brunetta E, et al. Early predictors of clinical deterioration in a cohort of 239 patients hospitalized for COVID-19 infection in Lombardy, Italy. J Clin Med 2020;9:1548. doi:10.3390/ jcm9051548

36 Colaneri M, Sacchi P, Zuccaro V, et al. Clinical characteristics of coronavirus disease (COVID-19) early findings from a teaching hospital in Pavia, North Italy, 21 to 28 February 2020. Euro Surveill 2020;25.

37 Covino M, De Matteis G, Santoro M. Clinical characteristics and prognostic factors in COVID-19 patients aged $>=80$ years. Geriat Gerontol Int 2020.

38 Cummings MJ, Baldwin MR, Abrams D. Epidemiology, clinical course, and outcomes of critically ill adults with COVID-19 in New York City: a prospective cohort study. medRxiv 2020.

39 D'Silva KM, Serling-Boyd N, Wallwork R, et al. Clinical characteristics and outcomes of patients with coronavirus disease 2019 (COVID-19) and rheumatic disease: a comparative cohort study from a US 'hot spot'. Ann Rheum Dis 2020;79:1156-62.

40 Docherty AB, Harrison EM, Green CA, et al. Features of 20133 UK patients in hospital with covid-19 using the ISARIC WHO Clinical Characterisation Protocol: prospective observational cohort study. BMJ 2020;369:m1985

41 El-Boghdadly K, Wong DJN, Owen R, et al. Risks to healthcare workers following tracheal intubation of patients with COVID-19: a prospective international multicentre cohort study. Anaesthesia 2020;75:1437-47.

42 Giacomelli A, Ridolfo AL, Milazzo L, et al. 30-day mortality in patients hospitalized with COVID-19 during the first wave of the Italian epidemic: a prospective cohort study. Pharmacol Res 2020;158:104931.

43 Gold JAW, Wong KK, Szablewski CM, et al. Characteristics and clinical outcomes of adult patients hospitalized with COVID-19 - Georgia, March 2020. MMWR Morb Mortal Wkly Rep 2020:69:545-50.

44 Hajifathalian K, Krisko T, Mehta A. Gastrointestinal and hepatic manifestations of 2019 novel coronavirus disease in a large cohort of infected patients from New York: clinical implications. Gastroenterol 2020

45 Hajifathalian K, Kumar S, Newberry C, et al. Obesity is associated with worse outcomes in COVID-19: analysis of early data from New York City. Obesity 2020;28:1606-12.

46 Hamer M, Kivimäki M, Gale CR, et al. Lifestyle risk factors, inflammatory mechanisms, and COVID-19 hospitalization: a community-based cohort study of 387,109 adults in UK. Brain Behav Immun 2020;87:184-7.

47 Hur K, Price CPE, Gray EL. Factors associated with intubation and prolonged intubation in hospitalized patients with COVID-19. JAMA Otolaryngol Head Neck Surg 2020.

48 Imam Z, Odish F, Gill I, et al. Older age and comorbidity are independent mortality predictors in a large cohort of 1305 COVID-19 patients in Michigan, United States. J Intern Med 2020;288:469-76.

49 Kalligeros M, Shehadeh F, Mylona EK, et al. Association of obesity with disease severity among patients with coronavirus disease 2019 . Obesity 2020;28:1200-4

50 Klang E, Kassim G, Soffer S, et al. Severe obesity as an independent risk factor for COVID-19 mortality in hospitalized patients younger than 50. Obesity 2020;28:1595-9.

51 Lassale C, Gaye B, Hamer M. Ethnic disparities in hospitalisation for COVID-19 in England: the role of socioeconomic factors, mental health, and inflammatory and pro-inflammatory factors in a community-based cohort study. Brain Behav Immun 2020.

52 Okoh AK, Sossou C, Dangayach NS, et al. Coronavirus disease 19 in minority populations of Newark, New Jersey. Int J Equity Health 2020;19:93

53 Palaiodimos L, Kokkinidis DG, Li W, et al. Severe obesity, increasing age and male sex are independently associated with worse inhospital outcomes, and higher in-hospital mortality, in a cohort of patients with COVID-19 in the Bronx, New York. Metabolism 2020;108:154262.

54 Patel AP, Paranjpe MD, Kathiresan NP, et al. Race, socioeconomic deprivation, and hospitalization for COVID-19 in English participants of a national Biobank. Int J Equity Health 2020;19.

55 Perez-Guzman PN, Daunt A, Mukherjee S. Report 17: clinical characteristics and predictors of outcomes of hospitalized patients 
with COVID-19 in a London NHS trust: a retrospective cohort study. London: Imperial College, 2020.

56 Petrilli CM, Jones SA, Yang J, et al. Factors associated with hospital admission and critical illness among 5279 people with coronavirus disease 2019 in New York City: prospective cohort study. BMJ 2020;369:m1966.

57 Piano S, Dalbeni A, Vettore E, et al. Abnormal liver function tests predict transfer to intensive care unit and death in COVID-19. Liver Int 2020;40:2394-406.

58 Price-Haywood EG, Burton J, Fort D, et al. Hospitalization and mortality among black patients and white patients with COVID-19. NEJM2020.

59 Shah V, Ko Ko T, Zuckerman M, et al. Poor outcome and prolonged persistence of SARS-CoV-2 RNA in COVID-19 patients with haematological malignancies; King's College Hospital experience. $\mathrm{Br}$ $\checkmark$ Haematol 2020;190.
60 Singh S, Khan A. Clinical characteristics and outcomes of COVID-19 among patients with pre-existing liver disease in United States: a multi-center research network study. Gastroenterol 2020.

61 Singh S, Khan A, Chowdhry M, et al. Risk of severe coronavirus disease 2019 in patients with inflammatory bowel disease in the United States: a multicenter research network study. Gastroenterology 2020;159:1575-8.

62 Violi F, Cangemi R, Romiti GF, et al. Is albumin predictor of mortality in COVID-19? Antioxid Redox Signal 2020.

63 England PH. Disparities in the risk and outcomes of COVID-19. London, UK: Public Health England, 2020.

64 Waffenschmidt S, Knelangen M, Sieben W, et al. Single screening versus conventional double screening for study selection in systematic reviews: a methodological systematic review. BMC Med Res Methodol 2019;19:132. 